Discrete Comput Geom 27:49-63 (2002)

DOI: $10.1007 / \mathrm{s} 00454-001-0051-\mathrm{x}$

\title{
A Combinatorial Study of Multiplexes and Ordinary Polytopes
}

\author{
M. M. Bayer, A. M. Bruening, ${ }^{*}$ and J. D. Stewart ${ }^{\dagger}$ \\ Department of Mathematics, University of Kansas, \\ Lawrence, KS 66045-7567, USA \\ bayer@math.ukans.edu
}

\begin{abstract}
Bisztriczky defines a multiplex as a generalization of a simplex, and an ordinary polytope as a generalization of a cyclic polytope. This paper presents results concerning the combinatorics of multiplexes and ordinary polytopes. The flag vector of the multiplex is computed, and shown to equal the flag vector of a many-folded pyramid over a polygon. Multiplexes, but not other ordinary polytopes, are shown to be elementary. It is shown that all complete subgraphs of the graph of a multiplex determine faces of the multiplex. The toric $h$-vectors of the ordinary five-dimensional polytopes are given. Graphs of ordinary polytopes are studied. Their chromatic numbers and diameters are computed, and they are shown to be Hamiltonian.
\end{abstract}

\section{Introduction}

A convex polytope is the convex hull of a finite set of points in $\mathbf{R}^{d}$. A $d$-dimensional convex polytope has proper faces of every dimension from zero (the vertices) to $d-1$ (the facets). These faces, along with the empty set and the polytope itself (the "improper faces"), ordered by inclusion, form a lattice. The properties of the face lattice are known as the combinatorial properties of the polytope. Of particular interest are the number of faces of each dimension (the $f$-vector), the graph consisting of the vertices and edges, and the polyhedral and simplicial complexes that subdivide the polytope. (The latter are not strictly speaking combinatorial. Different polytopes with the same face lattice can have different geometric subdivisions.)

The greatest progress in understanding combinatorial properties of convex polytopes has been made for the special class of simplicial polytopes. A simplex is the convex hull of affinely independent points. A polytope is simplicial if all its (proper) faces are simplices. A milestone was the characterization of the $f$-vectors of simplicial polytopes,

\footnotetext{
* Current address: 1502 Cypress Drive, Greenwood, MO 64034, USA

$\dagger$ Current address: 5381 Brook Way \#6, Columbia, MD 21044, USA.
} 
conjectured by McMullen [11], and proved by Billera and Lee [4] and Stanley [13]. In that work as well as research on triangulations of simplicial polytopes, the cyclic polytopes play a special role. These are simplicial polytopes, with vertices chosen as points on the moment curve, $\left\{\left(t, t^{2}, t^{3}, \ldots, t^{d}\right): t \in \mathbf{R}\right\}$. The combinatorial structure of the cyclic polytope depends only on the number, not the placement, of the points on the moment curve.

The combinatorial study of general polytopes is hampered by the difficulty in generating random combinatorial structures. A polytope formed by choosing points at random and taking their convex hull is generally simplicial. An alternative construction, of intersecting a randomly chosen set of hyperplanes, produces simple polytopes, which have face lattices dual to simplicial polytopes. Various geometric operations (for example, pyramiding and truncation) can be performed on polytopes to produce nonsimplex faces, but the effect of these on the face lattice is quite controlled.

Bisztriczky [7] defines a class of nonsimplicial polytopes called ordinary polytopes. The faces of ordinary polytopes are multiplexes, which generalize the simplex in a combinatorial way, but are generally not simplicial. A special case of the ordinary polytope is the cyclic polytope. Thus, ordinary polytopes hold out promise of playing an important role in the combinatorial study of nonsimplicial polytopes. In this paper we continue the study of multiplexes and ordinary polytopes begun by Bisztriczky [6], [7] and Dinh [8].

\section{Multiplexes}

Bisztriczky defines a multiplex as a generalization of a simplex.

Definition 1 [6]. A multiplex is a polytope with an ordered list of vertices, $x_{0}, x_{1}, \ldots$, $x_{n}$, with facets $F_{0}, F_{1}, \ldots, F_{n}$ given by

$$
F_{i}=\operatorname{conv}\left\{x_{i-d+1}, x_{i-d+2}, \ldots, x_{i-1}, x_{i+1}, x_{i+2}, \ldots, x_{i+d-1}\right\}
$$

with the conventions that $x_{i}=x_{0}$ if $i<0$, and $x_{i}=x_{n}$ if $i>n$.

Note that if $n=d$, then the multiplex is a simplex. It is easy to check that for $n=d+1$, the multiplex is a $(d-2)$-fold pyramid over a quadrilateral with vertex set $\left\{x_{0}, x_{1}, x_{d}, x_{d+1}\right\}$. Multiplexes are not all pyramids, however. Every polygon (two-dimensional polytope) is a multiplex with an appropriate ordering of vertices. Following are important results about multiplexes by Bisztriczky. For $n \geq d \geq 2$, let $M^{d, n}$ be the $d$-dimensional multiplex with $n+1$ vertices.

\section{Theorem 2.1 [6].}

1. $M^{d, n}$ exists for every $d$ and $n$ with $n \geq d \geq 2$.

2. Every multiplex is self-dual.

3. Every face and every quotient of a multiplex is a multiplex.

4. The number of $i$-dimensional faces of $M^{d, n}$ is $\left(\begin{array}{c}d+1 \\ i+1\end{array}\right)+(n-d)\left(\begin{array}{c}d-1 \\ i\end{array}\right)$.

The $f$-vector of $M^{d, n}$ is the same as the $f$-vector of a certain pyramid over a polygon. This fact extends to the "flag vector" of the multiplex. A chain of faces, $\emptyset \subset F_{1} \subset F_{2} \subset$ 
$\cdots \subset F_{r} \subset P$ is an $S$-flag if $S=\left\{\operatorname{dim} F_{1}, \operatorname{dim} F_{2}, \ldots, \operatorname{dim} F_{r}\right\}$. The number of $S$-flags of a polytope $P$ is written $f_{S}(P)$, and the length $2^{d}$ vector $\left(f_{S}(P)\right)_{S \subseteq\{0,1, \ldots, d-1\}}$ is the flag vector of $P$. In [15] Stanley observed that the flag vectors of multiplexes are flag vectors of products of face lattices of polygons. The following gives the flag vectors more precisely.

Theorem 2.2. The multiplex $M^{d, n}$ has the same flag vector as the $(d-2)$-fold pyramid over the $(n-d+3)$-gon. The common flag vector is given by

$$
\begin{aligned}
f_{S}= & \left(\begin{array}{c}
d+1 \\
s_{1}+1, s_{2}-s_{1}, \ldots, s_{r}-s_{r-1}, d-s_{r}
\end{array}\right) \\
& \times\left[1+\frac{n-d}{(d+1) d(d-1)} \sum_{j=1}^{r}\left(s_{j}+1\right)\left(s_{j+1}-s_{j}\right)\left(s_{j+1}-1\right)\right],
\end{aligned}
$$

where $S=\left\{s_{1}, s_{2}, \ldots, s_{r}\right\}, s_{1}<s_{2}<\cdots<s_{r}$, and $s_{r+1}=d$.

Proof. The flag vectors of the multiplexes and the $(d-2)$-fold pyramids can be computed from their common $f$-vector recursively, using only the hereditary property of the face lattices, shared by the two types of polytope. That is, every interval in the face lattice of a multiplex is the face lattice of a multiplex, and every interval in the face lattice of an iterated pyramid over a polygon is the face lattice of an iterated pyramid over a polygon.

Let $\mathcal{P}=\left\{Q^{d, n}: n \geq d \geq 2\right\}$ be a family of polytopes satisfying

1. For $0 \leq i \leq d-1$,

$$
f_{i}\left(Q^{d, n}\right)=\left(\begin{array}{c}
d+1 \\
i+1
\end{array}\right)+(n-d)\left(\begin{array}{c}
d-1 \\
i
\end{array}\right) .
$$

2. For any vertex $x$ of a polytope $Q^{d, n}$, the interval $\left[x, Q^{d, n}\right]$ is the face lattice of a polytope in $\mathcal{P}$ (if it is of rank three or greater).

The set of multiplexes is such a family; so is the set of iterated pyramids over polygons (letting $Q^{n, d}$ be the ( $d-2$ )-fold pyramid over the $(n-d+3)$-gon).

We calculate first $f_{0, i}\left(Q^{d, n}\right)$ for $1 \leq i \leq d-1$ :

$$
\begin{aligned}
f_{0, i}\left(Q^{d, n}\right) & =\sum_{x \text { vertex of } Q^{d, n}} f_{i-1}\left(\left[x, Q^{d, n}\right]\right) \\
& =\sum_{x \operatorname{vertex~of~} Q^{d, n}}\left(\begin{array}{l}
d \\
i
\end{array}\right)+\left(f_{0}\left(\left[x, Q^{d, n}\right]\right)-d\right)\left(\begin{array}{c}
d-2 \\
i-1
\end{array}\right) \\
& =(n+1)\left(\begin{array}{c}
d \\
i
\end{array}\right)-(n+1) d\left(\begin{array}{c}
d-2 \\
i-1
\end{array}\right)+\left(\begin{array}{c}
d-2 \\
i-1
\end{array}\right) \sum_{x \text { vertex of } Q^{d, n}} f_{0}\left(\left[x, Q^{d, n}\right]\right) \\
& =(n+1)\left(\begin{array}{c}
d \\
i
\end{array}\right)-(n+1) d\left(\begin{array}{c}
d-2 \\
i-1
\end{array}\right)+\left(\begin{array}{c}
d-2 \\
i-1
\end{array}\right) f_{0,1}\left(Q^{d, n}\right)
\end{aligned}
$$




$$
\begin{aligned}
= & (n+1)\left(\begin{array}{l}
d \\
i
\end{array}\right)-(n+1) d\left(\begin{array}{l}
d-2 \\
i-1
\end{array}\right)+\left(\begin{array}{l}
d-2 \\
i-1
\end{array}\right) 2 f_{1}\left(Q^{d, n}\right) \\
= & (n+1)\left(\begin{array}{l}
d \\
i
\end{array}\right)-(n+1) d\left(\begin{array}{l}
d-2 \\
i-1
\end{array}\right)+\left(\begin{array}{l}
d-2 \\
i-1
\end{array}\right) \\
& \times(d(d+1)+2(n-d)(d-1)) \\
= & (n+1)\left(\begin{array}{l}
d \\
i
\end{array}\right)+(d-2)(n-d)\left(\begin{array}{l}
d-2 \\
i-1
\end{array}\right) .
\end{aligned}
$$

This can be shown to agree with (1). We now show that all components of the flag vector of $Q^{d, n}$ are given by (1), by induction on $|S|$. Let $S=\left\{s_{1}, s_{2}, \ldots, s_{r}\right\}$ with $s_{1}<s_{2}<\cdots<s_{r}$.

$$
\begin{aligned}
& f_{S}\left(Q^{d, n}\right)=\sum_{F s_{r} \text {-face of } Q^{d, n}} f_{S \backslash\left\{s_{r}\right\}}(F) \\
& =\sum_{F s_{r} \text {-face of } Q^{d, n}} f_{S \backslash\left\{s_{r}\right\}}\left(Q^{s_{r}, f_{0}(F)-1}\right) \\
& =\sum_{F s_{r} \text {-face of } Q^{d, n}}\left(\begin{array}{c}
s_{r}+1 \\
s_{1}+1, s_{2}-s_{1}, \ldots, s_{r}-s_{r-1}
\end{array}\right) \\
& \times\left[1+\frac{f_{0}(F)-s_{r}-1}{\left(s_{r}+1\right) s_{r}\left(s_{r}-1\right)} \sum_{j=1}^{r-1}\left(s_{j}+1\right)\left(s_{j+1}-s_{j}\right)\left(s_{j+1}-1\right)\right] \\
& =\left(\begin{array}{c}
s_{r}+1 \\
s_{1}+1, s_{2}-s_{1}, \ldots, s_{r}-s_{r-1}
\end{array}\right) \\
& \times\left[1-\frac{s_{r}+1}{\left(s_{r}+1\right) s_{r}\left(s_{r}-1\right)} \sum_{j=1}^{r-1}\left(s_{j}+1\right)\left(s_{j+1}-s_{j}\right)\left(s_{j+1}-1\right)\right] \sum_{F s_{r} \text {-face of } Q^{d, n}} 1 \\
& +\left(\begin{array}{c}
s_{r}+1 \\
s_{1}+1, s_{2}-s_{1}, \ldots, s_{r}-s_{r-1}
\end{array}\right) \\
& \times\left[\frac{1}{\left(s_{r}+1\right) s_{r}\left(s_{r}-1\right)} \sum_{j=1}^{r-1}\left(s_{j}+1\right)\left(s_{j+1}-s_{j}\right)\left(s_{j+1}-1\right)\right] \sum_{F s_{r} \text {-face of } Q^{d, n}} f_{0}(F) \\
& =\left(\begin{array}{c}
s_{r}+1 \\
s_{1}+1, s_{2}-s_{1}, \ldots, s_{r}-s_{r-1}
\end{array}\right) \\
& \times\left[1-\frac{s_{r}+1}{\left(s_{r}+1\right) s_{r}\left(s_{r}-1\right)} \sum_{j=1}^{r-1}\left(s_{j}+1\right)\left(s_{j+1}-s_{j}\right)\left(s_{j+1}-1\right)\right] f_{s_{r}}\left(Q^{d, n}\right)
\end{aligned}
$$




$$
\begin{aligned}
& +\left(\begin{array}{c}
s_{r}+1 \\
s_{1}+1, s_{2}-s_{1}, \ldots, s_{r}-s_{r-1}
\end{array}\right) \\
& \times\left[\frac{1}{\left(s_{r}+1\right) s_{r}\left(s_{r}-1\right)} \sum_{j=1}^{r-1}\left(s_{j}+1\right)\left(s_{j+1}-s_{j}\right)\left(s_{j+1}-1\right)\right] f_{0, s_{r}}\left(Q^{d, n}\right) \\
& =\left(\begin{array}{c}
s_{r}+1 \\
s_{1}+1, s_{2}-s_{1}, \ldots, s_{r}-s_{r-1}
\end{array}\right) \\
& \times\left[1-\frac{s_{r}+1}{\left(s_{r}+1\right) s_{r}\left(s_{r}-1\right)} \sum_{j=1}^{r-1}\left(s_{j}+1\right)\left(s_{j+1}-s_{j}\right)\left(s_{j+1}-1\right)\right] \\
& \times\left[\left(\begin{array}{c}
d+1 \\
s_{r}+1
\end{array}\right)+(n-d)\left(\begin{array}{c}
d-1 \\
s_{r}
\end{array}\right)\right] \\
& +\left(\begin{array}{c}
s_{r}+1 \\
s_{1}+1, s_{2}-s_{1}, \ldots, s_{r}-s_{r-1}
\end{array}\right) \frac{1}{\left(s_{r}+1\right) s_{r}\left(s_{r}-1\right)} \\
& \times \sum_{j=1}^{r-1}\left(s_{j}+1\right)\left(s_{j+1}-s_{j}\right)\left(s_{j+1}-1\right) \\
& \times\left[(n+1)\left(\begin{array}{l}
d \\
s_{r}
\end{array}\right)+(d-2)(n-d)\left(\begin{array}{l}
d-2 \\
s_{r}-1
\end{array}\right)\right] .
\end{aligned}
$$

Algebraic manipulation gives (1). The formula for the flag vector thus holds for both multiplexes and iterated pyramids over polygons.

The toric $h$-vector of a polytope is a length $d+1$ vector whose components are linear functions of the flag vector. When the polytope has rational vertices, the toric $h$-vector is the sequence of middle perversity intersection homology Betti numbers of the associated toric variety. (The multiplex has a rational realization.) The toric $h$-vector for Eulerian posets (including face lattices of polytopes) is defined by Stanley [14]; see [3] for formulas in terms of the flag vector. Since it depends only on the flag vector, the toric $h$-vector of the multiplex is the same as the toric $h$-vector of the pyramid over the appropriate polygon. The $h$-vector of $M^{d, n}$ is thus

$$
h\left(M^{d, n}\right)=(1,1,1, \ldots, 1,1)+(n-d)(0,1,1, \ldots, 1,0) .
$$

A $d$-dimensional polytope $P$ is elementary if and only if the second and third entries in the toric $h$-vector are equal. In terms of the flag vector, this says $f_{02}(P)-3 f_{2}(P)+$ $f_{1}(P)-d f_{0}(P)+\left(\begin{array}{c}d+1 \\ 2\end{array}\right)=0$. (See [10].)

Corollary 2.3. For every $n \geq d \geq 2$, the multiplex $M^{d, n}$ is an elementary polytope.

Every face of a multiplex is a multiplex, but not all multiplexes are faces of higherdimensional multiplexes. 
Proposition 2.4. The multiplex $M^{d, n}$ is a proper face of some multiplex if and only if $d \leq n \leq 2 d-1$.

Proof. Assume $M$ is a $d$-dimensional multiplex with $n+1$ vertices, and suppose $M$ is a proper face of some higher-dimensional multiplex $\hat{M}$. Then $M$ is a facet of a multiplex of dimension $d+1$, namely, any $(d+1)$-dimensional face of $\hat{M}$ containing $M$. According to the description of the facets in the definition of multiplex, $M$ has at most $2 d$ vertices. So $d+1 \leq n+1 \leq 2 d$.

To prove the converse, let $Q$ be the $(d+1)$-dimensional multiplex with $2 d+1$ vertices. The facets of $Q$ are

$$
F_{i}=\operatorname{conv}\left\{x_{i-d}, x_{i-d+1}, \ldots, x_{i-1}, x_{i+1}, x_{i+2}, \ldots, x_{i+d}\right\},
$$

for $0 \leq i \leq 2 d$. For $1 \leq i \leq d, F_{i}$ is a facet of $Q$ with $i+d$ vertices. Thus among the proper faces of $Q$ are the $d$-multiplexes with $n+1$ vertices for every $n, d \leq n \leq 2 d-1$.

Note that by Theorem 2.1, every polygon is a multiplex. However, Proposition 2.4 says that the two-dimensional faces of higher-dimensional multiplexes can only be triangles and quadrilaterals.

We turn now to the graphs of multiplexes.

Theorem 2.5 [6]. Let $M^{d, n}$ be the multiplex with vertex set $\left\{x_{0}, x_{1}, \ldots, x_{n}\right\}$. The edges of $M^{d, n}$ are

- $\operatorname{conv}\left\{x_{i}, x_{j}\right\}$, where $0 \leq i<j \leq n$ and $j-i \leq d-2$,

- $\operatorname{conv}\left\{x_{i}, x_{i+d}\right\}$, where $0 \leq i \leq n-d$,

- $\operatorname{conv}\left\{x_{0}, x_{d-1}\right\}$,

- $\operatorname{conv}\left\{x_{n-d+1}, x_{n}\right\}$.

There is a significant literature on the subject of reconstructing a polytope from its graph. For simplicial polytopes, the reconstruction problem can be phrased as the problem of deciding when the vertices of a complete subgraph span a face of the polytope. The latter problem also arises when studying triangulations of polytopes.

Theorem 2.6. Every complete subgraph of the graph of a multiplex is the graph of a face of the multiplex.

Proof. If $n=d$, then $M^{d, n}$ is a simplex, and the statement is true. Assume $n>d$. Let $G$ be the graph of the multiplex $M^{d, n}$. Let $X=\left\{x_{\ell_{0}}, x_{\ell_{1}}, \ldots, x_{\ell_{t}}\right\} \subseteq \operatorname{vert}\left(M^{d, n}\right)$ with $\ell_{0}<\ell_{1}<\cdots<\ell_{t}$ such that for all $i, j \in\{0,1, \ldots, t\},\left\{x_{\ell_{i}}, x_{\ell_{j}}\right\}$ is an edge of $G$.

Case 1 . If $\ell_{0}=0$, then $\ell_{t} \leq d$. Note that $\left\{x_{1}, x_{d}\right\}$ is not an edge of $G$, so it is not a subset of $X$. Thus $X$ is contained in either the facet $F_{0}$ or the facet $F_{1}$ of the multiplex $M^{d, n}$. Since both those facets are simplices, $\operatorname{conv}(X)$ is a simplex that is a face of $M^{d, n}$.

If $\ell_{t}=n$, the argument is similar.

Case 2. Assume $0<\ell_{0}<\ell_{t}<n$. Note that each vertex $x_{i}$ of $M^{d, n}$ is contained in exactly the facets $F_{i-d+1}, F_{i-d+2}, \ldots, F_{i-1}, F_{i+1}, F_{i+2}, \ldots, F_{i+d-1}$. Here we use the 
conventions that $F_{i}=F_{0}$ if $i<0$, and $F_{i}=F_{n}$ if $i>n$. (The self-duality of $M^{d, n}$ is expressed in the combinatorial description.) Thus the set $X$ is contained in the facet $F_{j}$ if and only if $\ell_{t}-d+1 \leq j \leq \ell_{0}+d-1$ and $j \notin\left\{\ell_{0}, \ell_{1}, \ldots, \ell_{t}\right\}$. (This statement is not true if $\ell_{0}=0$ or $\ell_{t}=n$.) Let $a=\max \left\{0, \ell_{t}-d+1\right\}$ and $b=\min \left\{n, \ell_{0}+d-1\right\}$. Then we can write the set $J=\left\{j: X \subset F_{j}\right\}$ as $J=\{a, a+1, \ldots, b\} \backslash\left\{\ell_{0}, \ell_{1}, \ldots, \ell_{t}\right\}$. The set of vertices in $\bigcap_{j \in J} F_{j}$ is exactly $X$, i.e., $\bigcap_{j \in J} F_{j}=\operatorname{conv}(X)$. To check this, we need to know that $\{a, b\} \subseteq J$. If $a=0$, then $a$ is in $J$. Assume $a \neq 0$; note that since $x_{\ell_{0}}$ and $x_{\ell_{t}}$ are assumed to be adjacent, either $\ell_{t}-d+1<\ell_{0}$ (so $a \in J$ ), or $\ell_{t}=\ell_{0}+d$. In the latter case, $a=\ell_{t}-d+1=\ell_{0}+1$. Since $x_{\ell_{0}+1}$ is not adjacent to $x_{\ell_{t}}=x_{\ell_{0}+d}, a=\ell_{0}+1 \notin\left\{\ell_{0}, \ell_{1}, \ldots, \ell_{t}\right\}$, so $a \in J$. Similarly, $b \in J$. Now suppose $i \notin\left\{\ell_{0}, \ell_{1}, \ldots, \ell_{t}\right\}$; we wish to show that $x_{i} \notin \bigcap_{j \in J} F_{j}$. If $i<a$, then $x_{i} \notin F_{b}$. If $i>b$, then $x_{i} \notin F_{a}$. If $a \leq i \leq b$, then $x_{i} \in \bigcap_{j \in J} F_{j}$ if and only if $i \in\left\{\ell_{0}, \ell_{1}, \ldots, \ell_{t}\right\}$. Thus $\bigcap_{j \in J} F_{j}=\operatorname{conv}(X)$.

Now observe that this argument is also valid for every subsequence of $\ell_{0}<\ell_{1}<$ $\cdots<\ell_{t}$. Thus, for every $Y \subseteq X, \operatorname{conv}(Y)$ is a face of $M^{d, n}$, and so also a face of $\operatorname{conv}(X)$. Therefore $\operatorname{conv}(X)$ is a simplex.

This last theorem enables us to count the number of faces that are simplices.

Proposition 2.7. For $n>d$, the number of $i$-dimensional simplex faces of the multiplex $M^{d, n}$ is

$$
\left(\begin{array}{l}
d+1 \\
i+1
\end{array}\right)-\left(\begin{array}{c}
d-3 \\
i-3
\end{array}\right)+(n-d)\left[\left(\begin{array}{c}
d-1 \\
i
\end{array}\right)-\left(\begin{array}{c}
d-3 \\
i-2
\end{array}\right)\right] .
$$

Proof. Count the number of complete subgraphs with vertex set $X=\left\{x_{\ell_{0}}, x_{\ell_{1}}, \ldots, x_{\ell_{i}}\right\}$, with $\ell_{0}<\ell_{1}<\cdots<\ell_{i}$.

- For $\ell_{0}=0$, the complete subgraphs are obtained by choosing $i$-element sets from $\{1,2, \ldots, d\}$ not containing the pair $\{1, d\}$, since $\left\{x_{1}, x_{d}\right\}$ is the only nonedge using these indices. There are $\left(\begin{array}{l}d \\ i\end{array}\right)-\left(\begin{array}{c}d-2 \\ i-2\end{array}\right)$ such complete subgraphs.

- For $1 \leq \ell_{0} \leq n-d-1$, the complete subgraphs are obtained by choosing $i$-element sets from $\left\{\ell_{0}+1, \ell_{0}+2, \ldots, \ell_{0}+d-2, \ell_{0}+d\right\}$ not containing the pair $\left\{\ell_{0}+1, \ell_{0}+d\right\}$. There are $\left(\begin{array}{c}d-1 \\ i\end{array}\right)-\left(\begin{array}{c}d-3 \\ i-2\end{array}\right)$ such complete subgraphs for each $\ell_{0}$, or $(n-d-1)\left[\left(\begin{array}{c}d-1 \\ i\end{array}\right)-\left(\begin{array}{c}d-3 \\ i-2\end{array}\right)\right]$ altogether.

- For $\ell_{0}=n-d$, the complete subgraphs are obtained by choosing $i$-element sets from $\{n-d+1, n-d+2, \ldots, n-2, n\}$. There are $\left(\begin{array}{c}d-1 \\ i\end{array}\right)$ such complete subgraphs.

- For $n-d+1 \leq \ell_{0} \leq n-i$, the complete subgraphs are obtained by choosing $i$-element sets from $\left\{\ell_{0}+1, \ell_{0}+2, \ldots, n-1, n\right\}$. There are $\left(\begin{array}{c}n-\ell_{0} \\ i\end{array}\right)$ such complete subgraphs.

Altogether the number of $i$-faces that are simplices is thus

$$
\begin{aligned}
\left(\begin{array}{c}
d \\
i
\end{array}\right)- & \left(\begin{array}{c}
d-2 \\
i-2
\end{array}\right)+(n-d-1)\left[\left(\begin{array}{c}
d-1 \\
i
\end{array}\right)-\left(\begin{array}{c}
d-3 \\
i-2
\end{array}\right)\right]+\left(\begin{array}{c}
d-1 \\
i
\end{array}\right)+\sum_{j=i}^{d-1}\left(\begin{array}{l}
j \\
i
\end{array}\right) \\
& =\left(\begin{array}{c}
d \\
i
\end{array}\right)-\left(\begin{array}{c}
d-2 \\
i-2
\end{array}\right)+(n-d)\left[\left(\begin{array}{c}
d-1 \\
i
\end{array}\right)-\left(\begin{array}{c}
d-3 \\
i-2
\end{array}\right)\right]+\left(\begin{array}{c}
d-3 \\
i-2
\end{array}\right)+\left(\begin{array}{c}
d \\
i+1
\end{array}\right)
\end{aligned}
$$




$$
=\left(\begin{array}{c}
d+1 \\
i+1
\end{array}\right)-\left(\begin{array}{c}
d-3 \\
i-3
\end{array}\right)+(n-d)\left[\left(\begin{array}{c}
d-1 \\
i
\end{array}\right)-\left(\begin{array}{c}
d-3 \\
i-2
\end{array}\right)\right] .
$$

Thus, the number of nonsimplex $i$-faces of $M^{d, n}$ is $\left(\begin{array}{c}d-3 \\ i-3\end{array}\right)+(n-d)\left(\begin{array}{l}d-3 \\ i-2\end{array}\right)$.

Bisztriczky describes certain quadrilateral two-faces of the multiplex.

Proposition 2.8 [6]. For $n>d \geq 3$, let $M^{d, n}$ be the multiplex with ordered list of vertices $x_{0}, x_{1}, \ldots, x_{n}$. Then for each $i, 0 \leq i \leq n-d-1, \operatorname{conv}\left\{x_{i}, x_{i+1}, x_{i+d}, x_{i+d+1}\right\}$ is a quadrilateral two-face with diagonals $\operatorname{conv}\left\{x_{i}, x_{i+d+1}\right\}$ and $\operatorname{conv}\left\{x_{i+1}, x_{i+d}\right\}$.

From the comment above, we know that $M^{d, n}$ has exactly $n-d$ nonsimplex two-faces. Bisztriczky's proposition accounts for all of them.

The following propositions have analogues for ordinary polytopes, and those are proved in Section 3. The odd-dimensional multiplexes are also ordinary polytopes. The proofs of Propositions 3.10-3.12 carry through for the even-dimensional multiplexes as well. The first is of special interest because Kalai [10] conjectures that all elementary $d$-polytopes are $(d+1)$-colorable.

Proposition 2.9. The chromatic number of the graph of the multiplex $M^{d, n}$ is $d$, if $n>d$, and $d+1$, if $n=d$ (in which case $M^{d, n}$ is the $d$-simplex).

Proposition 2.10. For every $n \geq d \geq 2$, the multiplex $M^{d, n}$ has a Hamiltonian cycle.

Proposition 2.11. For every $n \geq d \geq 2$, the multiplex $M^{d, n}$ has diameter $\lceil n / d\rceil$.

\section{Ordinary Polytopes}

Given an ordered set $V=\left\{x_{0}, x_{1}, \ldots, x_{n}\right\}$, a subset $Y \subseteq V$ is called a Gale subset if between any two elements of $V \backslash Y$ there is an even number of elements of $Y$. A polytope $P$ with ordered vertex set $V$ as above is a Gale polytope if the set of vertices of each facet is a Gale subset.

Definition 2 [7]. An ordinary polytope is a Gale polytope such that each facet is a multiplex with the induced order on the vertices.

The definition of ordinary polytope is due to Bisztriczky. His choice of the term "ordinary" stemmed from a belief that they arise by choosing vertices on a convex ordinary space curve, but this is not understood in dimensions higher than three. See [5] and [7].

The combinatorics of three-dimensional ordinary polytopes differs considerably from that of higher-dimensional ordinary polytopes. In this paper we consider only ordinary polytopes of dimension at least four. Bisztriczky [7] defines these as above, and proves a number of results on their combinatorics. However, it is Dinh [8] who proves their existence in Euclidean space. We use the following theorems of Bisztriczky and Dinh. 
Theorem 3.1 [7]. Let $P$ be an ordinary d-polytope with ordered vertices $x_{0}, x_{1}, \ldots$, $x_{n}$. Assume $n \geq d \geq 4$.

1. If $d$ is even, then $P$ is cyclic.

2. If $d$ is odd, then there exists an integer $k(d \leq k \leq n)$ such that the vertices sharing an edge with $x_{0}$ are exactly $x_{1}, x_{2}, \ldots, x_{k}$, and the vertices sharing an edge with $x_{n}$ are exactly $x_{n-1}, x_{n-2}, \ldots, x_{n-k}$.

The integer $k$ guaranteed by this theorem is called the characteristic of the ordinary polytope.

Theorem 3.2 [8]. For every $n \geq k \geq d=2 m+1 \geq 5$, there exists an ordinary $d$-polytope with $n+1$ vertices and characteristic $k$.

Theorem 3.3 [7]. Given a triple of integers $(n, k, d)$ with $d$ odd and $n \geq k \geq d \geq 5$, all ordinary d-polytopes with $n+1$ vertices and characteristic $k$ are combinatorially isomorphic.

If $P$ is an ordinary d-polytope with $n+1$ vertices and characteristic $k=n$, then $P$ is a cyclic polytope.

If $P$ is an ordinary d-polytope with characteristic $k=d$, then $P$ is a multiplex.

Write $P^{d, k, n}$ for the $d$-dimensional ordinary polytope with $n+1$ vertices and characteristic $k$. The Appendix gives the face lattice of $P^{5,7,9}$; it was computed using the software package Polymake [9].

We do not have a formula for the complete flag vector of $P^{d, k, n}$. However, Dinh [8] computes the $f$-vector of $P^{d, k, n}$, and here we compute $f_{02}$, and show that ordinary polytopes, other than multiplexes, are not elementary.

We use the following description of the facets of $P^{d, k, n}$, due to Dinh.

Theorem 3.4 [8]. Let $n, k, d$, and $m$ be integers such that $n \geq k \geq d=2 m+1 \geq 5$. Let $P^{d, k, n}$ be an ordinary $d$-polytope with characteristic $k$ and ordered vertices $x_{0}, x_{1}$, $\ldots, x_{n}$. Then the facets of $P^{d, k, n}$ are $\operatorname{conv}(X)$, where

$$
X=\left\{x_{i}, x_{i+1}, \ldots, x_{i+2 r-1}\right\} \cup Y \cup\left\{x_{i+k}, x_{i+k+1}, \ldots, x_{i+k+2 r-1}\right\},
$$

where $i \in \mathbf{Z}, 1 \leq r \leq m, Y$ is a paired $(d-2 r-1)$-element subset of $\left\{x_{i+2 r+1}, x_{i+2 r+2}\right.$, $\left.\ldots, x_{i+k-2}\right\}$, and $|X| \geq d$. Here a paired subset is one whose index set can be written as a disjoint union of pairs of consecutive integers.

By definition each of these facets is a multiplex with the induced vertex ordering. Note that each facet (a $(d-1)$-dimensional multiplex) has at most $d+2 m-1=2 d-2$ vertices. Thus, Proposition 2.4 generalizes to

The multiplex $M^{d, n}$ is a proper face of some ordinary polytope if and only if $d \leq n \leq$ $2 d-1$. 
The facets of ordinary polytopes are small, simplex-like polytopes. The duals of ordinary polytopes have facets with many vertices, however. It can be shown that each facet of the dual of an ordinary $(2 m+1)$-polytope of characteristic $k$ has at least $3\left(\begin{array}{c}k-m-3 \\ m-1\end{array}\right)$ vertices. This is the number of those facets of $P^{d, k, n}$ that contain the vertex $x_{1}$ and fit the description of Theorem 3.4 with $r=1$.

The two-dimensional faces of $P^{d, k, n}$ are exactly the two-dimensional faces of its facets. From Proposition 2.8 and the comment following it, we know all the nontriangular two-faces.

Proposition 3.5. Let $n, k, d$, and $m$ be integers such that $n \geq k \geq d=2 m+1 \geq 5$. Let $P^{d, k, n}$ be an ordinary $d$-polytope with characteristic $k$ and ordered vertices $x_{0}, x_{1}$, $\ldots, x_{n}$. The two-dimensional faces of $P^{d, k, n}$ that are not triangles are exactly the sets $\operatorname{conv}\left\{x_{i}, x_{i+1}, x_{i+k}, x_{i+k+1}\right\}$, for $0 \leq i \leq n-k-1$.

Proof. Dinh [8] proves that these are indeed two-faces of $P^{d, k, n}$, but does not show that they are the only nontriangular two-faces. Consider a facet $F$ as given in Theorem 3.4, and apply Proposition 2.8 (and the subsequent comment) to give all its nontriangular two-faces. Renumber the vertices of the facet $F$ as $z_{0}, z_{1}, \ldots$ Thus the $d-2 r-1$ elements of $Y$ are numbered $z_{2 r}$ through $z_{d-2}$, and $\left\{x_{i+k}, x_{i+k+1}, \ldots, x_{i+k+2 r-1}\right\}=$ $\left\{z_{d-1}, z_{d}, \ldots, z_{d+2 r-2}\right\}$. The quadrilateral faces in $F$ are of the form $\operatorname{conv}\left\{z_{j}, z_{j+1}, z_{j+d-1}\right.$, $\left.z_{j+d}\right\}$. Such a quadrilateral contains no element of $Y$, and is of the form $\operatorname{conv}\left\{x_{\ell}, x_{\ell+1}\right.$, $\left.x_{\ell+k}, x_{\ell+k+1}\right\}$, with $i \leq \ell \leq i+2 r-2$. Considering all the facets of $P^{d, k, n}, \ell$ can range from 0 to $n-k-1$.

Dinh also computes the $f$-vectors of ordinary polytopes. At the moment we need only $f_{1}$.

Proposition 3.6 [8]. Let $n, k, d$, and $m$ be integers such that $n \geq k \geq d=2 m+1 \geq 5$. Then

$$
f_{1}\left(P^{d, k, n}\right)=\left(\begin{array}{c}
k+1 \\
2
\end{array}\right)+(n-k)(k-1) \text {. }
$$

Proposition 3.7. For every $n \geq k \geq d=2 m+1 \geq 5$, the ordinary polytope $P^{d, k, n}$ is elementary if and only if $k=d$, in which case the ordinary polytope is itself a multiplex.

Proof. Proposition 3.5 implies that for the ordinary polytope $P^{d, k, n}, f_{02}\left(P^{d, k, n}\right)=$ $3 f_{2}\left(P^{d, k, n}\right)+(n-k)$. Write

$$
\beta=f_{02}\left(P^{d, k, n}\right)-3 f_{2}\left(P^{d, k, n}\right)+f_{1}\left(P^{d, k, n}\right)-d f_{0}\left(P^{d, k, n}\right)+\left(\begin{array}{c}
d+1 \\
2
\end{array}\right) .
$$

Then

$$
\beta=(n-k)+\left(\begin{array}{c}
k+1 \\
2
\end{array}\right)+(n-k)(k-1)-d(n+1)+\left(\begin{array}{c}
d+1 \\
2
\end{array}\right) .
$$


The ordinary polytope $P^{d, k, n}$ is elementary if and only if $\beta=0$. If $k \neq d$, then solving $\beta=0$ for $n$ gives $n=(k+d-1) / 2$. However, $(k+d-1) / 2 \leq(2 k-1) / 2<k \leq n$, so this is not possible. Thus $\beta=0$ implies $k=d$, i.e., the ordinary polytope is a multiplex. We have already seen that every multiplex is elementary.

By Dinh's construction [8] of ordinary polytopes, they can be realized as rational polytopes. It would be interesting to compute the toric $h$-vector of ordinary polytopes. The quantities above are enough for the case of dimension five.

Theorem 3.8. Let $n \geq k \geq 5$. The toric h-vector of $P^{5, k, n}$ is

$$
\left(1, n-4,\left(\begin{array}{c}
n-3 \\
2
\end{array}\right)-\left(\begin{array}{c}
n-k+1 \\
2
\end{array}\right),\left(\begin{array}{c}
n-3 \\
2
\end{array}\right)-\left(\begin{array}{c}
n-k+1 \\
2
\end{array}\right), n-4,1\right) .
$$

Among all 5-polytopes with $h_{1}=n-4$ (that is, $f_{0}=n+1$ ), the smallest possible $h_{2}$ is $n-4$, and this is achieved by the multiplex $M^{5, n}=P^{5,5, n}$. Among all 5-polytopes with $h_{1}=n-4$, the largest possible $h_{2}$ is $\left(\begin{array}{c}n-3 \\ 2\end{array}\right)$, and this is achieved by the cyclic polytope $P^{5, n, n}$. Thus, the toric $h$-vectors of $P^{5, k, n}$ are nicely distributed between the extreme toric $h$-vectors having $h_{1}=n-4$. The formula for $h_{2}$ generalizes for ordinary polytopes of higher (odd) dimension:

$$
h_{2}\left(P^{d, k, n}\right)=\left(\begin{array}{c}
n-d+2 \\
2
\end{array}\right)-\left(\begin{array}{c}
n-k+1 \\
2
\end{array}\right)
$$

note that $\left(\begin{array}{c}n-d+2 \\ 2\end{array}\right)$ is $h_{2}$ for the cyclic $d$-polytope with $n+1$ vertices.

The description of the graph of a multiplex extends naturally to ordinary polytopes.

Theorem 3.9. For $n \geq k \geq d=2 m+1 \geq 5$, let $P^{d, k, n}$ be the ordinary polytope with vertex set $\left\{x_{0}, x_{1}, \ldots, x_{n}\right\}$. The edges of $P^{\overline{d, k, n}}$ are

- $\operatorname{conv}\left\{x_{i}, x_{j}\right\}$, where $0 \leq i<j \leq n$ and $j-i \leq k-2$,

- $\operatorname{conv}\left\{x_{i}, x_{i+k}\right\}$, where $0 \leq i \leq n-k$,

- $\operatorname{conv}\left\{x_{0}, x_{k-1}\right\}$,

- $\operatorname{conv}\left\{x_{n-k+1}, x_{n}\right\}$.

Proof. We consider the pairs not listed in the statement of the theorem. These fall into two categories. First are those pairs $\left\{x_{i}, x_{j}\right\}$ with $j-i \geq k+1$. From Theorem 3.4 every facet containing $x_{i}$ and $x_{j}$, with $j-i \geq k+1$, also contains the nonempty set of vertices $\left\{x_{i+k}, x_{i+k+1}, \ldots, x_{j-1}\right\}$. Thus, $\operatorname{conv}\left\{x_{i}, x_{j}\right\}$ is not a face (edge) of $P^{d, k, n}$. The number of these pairs is

$$
\sum_{i=0}^{n-k-1}(n-k-i)=\sum_{\ell=1}^{n-k} \ell=\left(\begin{array}{c}
n-k+1 \\
2
\end{array}\right) .
$$

The other pairs not listed are $\left\{x_{i}, x_{j}\right\}$ with $j-i=k-1, i \neq 0$, and $j \neq n$. For such pairs $\operatorname{conv}\left\{x_{i}, x_{j}\right\}$ is not an edge of $P^{d, k, n}$, because it is a diagonal of a two-dimensional 
face as described in Proposition 3.5. The number of these pairs is $n-k$. The number of pairs listed in the statement of the theorem is thus

$$
\left(\begin{array}{c}
n+1 \\
2
\end{array}\right)-\left(\begin{array}{c}
n-k+1 \\
2
\end{array}\right)-(n-k)=\left(\begin{array}{c}
k+1 \\
2
\end{array}\right)+(n-k)(k-1)
$$

which is the number of edges of $P^{d, k, n}$, as computed by Dinh (Proposition 3.6). So all the listed pairs are edges of $P^{d, k, n}$.

Note that the graphs of ordinary polytopes are dimensionally ambiguous. For every odd $d$ and $d^{\prime}$ between 5 and $k$, the graphs of $P^{d, k, n}$ and $P^{d^{\prime}, k, n}$ are isomorphic.

Proposition 3.10. For every $n \geq k \geq d=2 m+1 \geq 5$, the chromatic number of the graph of the ordinary polytope $P^{d, k, n}$ is $k$, if $n>k$, and $k+1$, if $n=k$ (in which case $P^{d, k, n}$ is a cyclic polytope).

Proof. If $n=k$, then $P=P^{d, k, n}$ is a cyclic polytope, and the graph of $P$ is the complete graph on $k+1$ vertices, so its chromatic number is $k+1$. So assume $n>k$. Let $G$ be the graph of $P$, with vertex set $\left\{x_{0}, x_{1}, \ldots, x_{n}\right\}$. Assign colors from the set $\{0,1, \ldots, k-1\}$ to the vertices of $G$ as follows:

$$
\lambda\left(x_{i}\right)= \begin{cases}k-1 & \text { if } \quad i=0 \quad \text { or } \quad i=n, \\ i \bmod (k-1) & \text { if } \quad 1 \leq i \leq n-1 .\end{cases}
$$

Since $x_{0}$ and $x_{n}$ are not adjacent in $G$, every edge containing $x_{0}$ or $x_{n}$ is assigned two different colors. If $i<j$ and $j-i \leq k-2$, or $j-i=k$, then $j \not \equiv i(\bmod (k-1))$, so $\lambda\left(x_{j}\right) \neq \lambda\left(x_{i}\right)$. Thus adjacent vertices have different colors, so $\lambda$ gives a proper $k$ coloring of $G$. Now $G$ contains a complete subgraph on the vertex set $\left\{x_{0}, x_{1}, \ldots, x_{k-1}\right\}$, so the chromatic number of $G$ is $k$.

Proposition 3.11. For every $n \geq k \geq d=2 m+1 \geq 5$, the ordinary polytope $P^{d, k, n}$ has a Hamiltonian cycle.

Proof. If $n$ is odd, the vertex sequence, $x_{0}, x_{2}, x_{4}, \ldots, x_{n-1}, x_{n}, x_{n-2}, x_{n-4}, \ldots, x_{3}, x_{1}$, $x_{0}$, gives a Hamiltonian cycle. If $n$ is even, the vertex sequence, $x_{0}, x_{2}, x_{4}, \ldots, x_{n-2}, x_{n}$, $x_{n-1}, x_{n-3}, \ldots, x_{3}, x_{1}, x_{0}$, gives a Hamiltonian cycle.

Proposition 3.12. For every $n \geq k \geq d=2 m+1 \geq 5$, the ordinary polytope $P^{d, k, n}$ has diameter $\lceil n / k\rceil$.

Proof. For $i<j$, the vertices $x_{i}$ and $x_{j}$ are adjacent if $j-i \leq k-2$ or if $j-i=k$. In addition $x_{0}$ and $x_{k-1}$ are adjacent, and $x_{n}$ and $x_{n-k+1}$ are adjacent. So usually $x_{i}$, $x_{i+k}, x_{i+2 k}, \ldots, x_{i+t k}, x_{j}$, with $t=\lfloor(j-i-1) / k\rfloor$, gives an $x_{i}-x_{j}$ path of length $t+1 \leq\lceil n / k\rceil$. This is valid as long as $j-i \not \equiv-1(\bmod k)$. If $j-i \equiv-1(\bmod k)$, then $x_{i}, x_{i+k}, x_{i+2 k}, \ldots, x_{i+t k}, x_{i+t k+1}, x_{j}$, with $t=\lfloor(j-i) / k\rfloor=(j-i-k+1) / k$, gives 
a path of length $t+2$. If $j-i<n-1$, then $t+2=(j-i+k+1) / k \leq\lceil n / k\rceil$. The remaining cases are the paths $x_{0}, x_{k-1}, x_{2 k-1}, \ldots, x_{n-1}$ and $x_{1}, x_{k+1}, x_{2 k+1}, \ldots, x_{n+1-k}$, $x_{n}$, if $n \equiv 0(\bmod k)$, and $x_{0}, x_{k}, x_{2 k}, \ldots, x_{t k}, x_{n}$, with $t=\lfloor n / k\rfloor$, if $n \equiv-1(\bmod k)$. These are all of length $\lceil n / k\rceil$. Clearly, the $x_{0}-x_{n}$ path given is the shortest $x_{0}-x_{n}$ path. So the diameter is exactly $\lceil n / k\rceil$.

By Theorem 3.3 the class of ordinary polytopes includes the odd-dimensional cyclic polytopes. Cyclic polytopes have played an important role in the combinatorial study of simplicial polytopes (e.g., in [11]), and, more recently, in the study of triangulations of polytopes (e.g., in [12]). Cyclic polytopes are neighborly, that is, every $\lfloor d / 2\rfloor$-element set of vertices is the vertex set of a face of the polytope. In particular, the graph of a neighborly $d$-polytope for $d \geq 4$ is the complete graph. Thus no ordinary polytopes other than the cyclic polytopes are neighborly. A generalization of neighborliness is studied in [1]. A polytope is weakly neighborly if every set of $k+1$ vertices is contained in a face of dimension at most $2 k$, for all $k$. It is natural to ask, then, if ordinary polytopes are weakly neighborly. The answer is no, almost always. If $n \geq k+2$, then $x_{0}$ and $x_{n}$ are not on a common two-face of the ordinary polytope $P^{d, k, n}$. For $n=k+1, k>d \geq 5$ ( $d$ odd), $P^{d, k, n}$ is not weakly neighborly; for example, $\left\{x_{1}, x_{3}, x_{5}\right\}$ is not contained in a facet of $P^{5,6,7}$. If $n=d+2$, then $x_{0}$ and $x_{n}$ are not on a common two-face of the multiplex $M^{d, n}$. The only weakly neighborly ordinary polytopes are the cyclic polytopes and the multiplexes $M^{d, d+1}$, which are $(d-2)$-fold pyramids over quadrilaterals. Theorem 2.6 says that every complete subgraph of the graph of a multiplex is the graph of a face of the multiplex. This fails in general for ordinary polytopes.

We turn now to the $f$-vectors of ordinary polytopes. These are computed by Dinh.

Theorem 3.13 [8]. Let $n \geq k \geq d=2 m+1 \geq 5$. The number of $i$-dimensional faces of the ordinary polytope $P^{\overline{d, k}, n}$ is

$$
f_{i}\left(P^{d, k, n}\right)=\varphi_{i}(d, k)+(n-k) c_{i}(d, k),
$$

where $\varphi_{i}(d, k)$ is the number of $i$-faces of the cyclic $d$-polytope with $k+1$ vertices,

$$
\begin{aligned}
& \varphi_{i}= \begin{cases}\left(\begin{array}{l}
k+1 \\
i+1
\end{array}\right) & \text { for } \quad 0 \leq i \leq m-1, \\
\sum_{j=0}^{m}\left[\left(\begin{array}{c}
j \\
d-1-i
\end{array}\right)+\left(\begin{array}{c}
d-j \\
d-1-i
\end{array}\right)\right]\left(\begin{array}{c}
k-d+j \\
j
\end{array}\right) & \text { for } \quad m \leq i \leq d-1,\end{cases} \\
& \text { and } c_{i}(d, k)=f_{i}\left(P^{d, k, n+1}\right)-f_{i}\left(P^{d, k, n}\right) \text { is given by } \\
& 1 . \\
& c_{i}=\left(\begin{array}{c}
k-1 \\
i
\end{array}\right), \quad \text { for } \quad 1 \leq i<m, \\
& 2 . \\
& c_{m}=\left(\begin{array}{c}
k-1 \\
m
\end{array}\right)-\left(\begin{array}{c}
k-2-m \\
m
\end{array}\right) \text {, }
\end{aligned}
$$


3.

$$
\begin{aligned}
c_{i}= & \sum_{j=i-m}^{\lfloor i / 2\rfloor}(2 N(k-1, j, i)-N(k-2, j, i)) \\
& -\sum_{j=i-m}^{\lfloor(i-1) / 2\rfloor} N(k-3, j, i-1) \\
& -\sum_{j=i-m-1}^{\lfloor(i-2) / 2\rfloor} N(k-3, j, i-2) \\
& -\sum_{r=0}^{i-m} N(k-3-2 r, i-m-r, i-2 r),
\end{aligned}
$$

for $m<i<2 m=d-1$, and

4.

$$
c_{d-1}=c_{2 m}=\left(\begin{array}{c}
k-2-m \\
m-1
\end{array}\right) \text {. }
$$

Here

$$
N(s, t, u)=\left(\begin{array}{c}
u-t \\
t
\end{array}\right)\left(\begin{array}{c}
s-u+t \\
u-t
\end{array}\right)+\left(\begin{array}{c}
u-1-t \\
t
\end{array}\right)\left(\begin{array}{l}
s-u+t \\
u-1-t
\end{array}\right)
$$

Thus, for fixed $d$ and $k$, the $f$-vectors of the ordinary polytopes $P^{d, k, n}$ lie on a line.

Conjecture 3.14. Let $d$ be an odd integer, $d \geq 5$. The set of $f$-vectors of all ordinary $d$-polytopes spans the Euler hyperplane (given by $\left.\sum_{i=0}^{d-1}(-1)^{i} f_{i}=2\right)$. A spanning set consists of the ordinary polytopes $P^{d, d+\lfloor i / 2\rfloor, d+i}$, for $1 \leq i \leq d$.

The conjecture has been verified on computer for odd $d, 5 \leq d \leq 37$.

The flag vectors of ordinary polytopes satisfy many linear relations that do not hold for all polytopes, however. Most of these relations hold more generally for multiplicial polytopes, polytopes all of whose proper faces are multiplexes. We will show in [2] that the set of flag vectors of multiplicial $d$-polytopes is of (linear) dimension at most $2 d-3$, while the set of flag vectors of ordinary $d$-polytopes ( $d$ odd) is of dimension at most $d$.

\section{Appendix}

Here is the face lattice of the ordinary polytope $P^{5,7,9}$. Faces are listed by their sets of vertices, from the vertex set $\{0,1, \ldots, 9\}$. The $f$-vector of this polytope is $f\left(P^{5,7,9}\right)=$ $(10,40,76,70,26)$.

Facets:

01234, 01245, 01256, 02345, 02356, 02367, 03456, 03467, 04567, 23459,

23569, 23679, 34569, 34679, 34789, 45679, 45789, 56789, 013478,

$014578,015678,123489,124589,125689,0123789,0126789$ 
Three-dimensional faces:

0123, 0124, 0125, 0126, 0134, 0145, 0156, 0234, 0235, 0236, 0237, 0245,

0256, 0267, 0345, 0346, 0347, 0356, 0367, 0456, 0457, 0467, 0567, 1234,

$1245,1256,1348,1458,1568,2345,2349,2356,2359,2367,2369,2379$,

2459, 2569, 2679, 3456, 3459, 3467, 3469, 3478, 3479, 3489, 3569, 3679,

$3789,4567,4569,4578,4579,4589,4679,4789,5678,5679,5689,5789$,

6789, 01378, 01478, 01578, 01678, 12389, 12489, 12589, 12689, 012789

Two-dimensional faces:

012, 013, 014, 015, 016, 023, 024, 025, 026, 027, 034, 035, 036, 037,

045, 046, 047, 056, 057, 067, 123, 124, 125, 126, 134, 138, 145, 148,

$156,158,168,234,235,236,237,239,245,249,256,259,267,269$,

$279,345,346,347,348,349,356,359,367,369,378,379,389,456$,

$457,458,459,467,469,478,479,489,567,568,569,578,579,589$,

$678,679,689,789,0178,1289$

Edges:

$01,02,03,04,05,06,07,12,13,14,15,16,18,23,24,25,26,27$,

$29,34,35,36,37,38,39,45,46,47,48,49,56,57,58,59,67,68$,

$69,78,79,89$

\section{References}

1. Margaret M. Bayer, Equidecomposable and weakly neighborly polytopes, Israel J. Math. 81 (1993), 301320.

2. Margaret M. Bayer, Flag vectors of ordinary polytopes, in preparation.

3. Margaret M. Bayer and Richard Ehrenborg, The toric $h$-vectors of partially ordered sets, Trans. Amer. Math. Soc. 352 (2000), 4515-4531.

4. Louis J. Billera and Carl W. Lee, A proof of the sufficiency of McMullen's conditions for $f$-vectors of simplicial polytopes, J. Combin. Theory Ser. A 31 (1981), 237-255.

5. Tibor Bisztriczky, Ordinary 3-polytopes, Geom. Dedicata 52 (1994), 129-142.

6. Tibor Bisztriczky, On a class of generalized simplices, Mathematika 43 (1996), 274-285.

7. Tibor Bisztriczky, Ordinary (2m+1)-polytopes, Israel J. Math. 102 (1997), 101-123.

8. Thi Ngoc Dinh, Ordinary Polytopes, Ph.D. thesis, The University of Calgary, Calgary, Alberta, 1999.

9. Ewgenij Gawrilow and Michael Joswig, Polymake: a framework for analyzing convex polytopes, in Polytopes - Combinatorics and Computation (Oberwolfach 1997), pp. 43-74. Birkhäuser, Basel, 2000.

10. Gil Kalai, Some aspects of the combinatorial theory of convex polytopes, in Polytopes: Abstract, Convex, and Computational, pp. 205-229. NATO Advanced Science Institutes Series C, Vol. 440. Kluwer, Dordrecht, 1994.

11. Peter McMullen, The maximum number of faces of a convex polytope, Mathematika 17 (1970), 179-184.

12. Jörg Rambau and Francisco Santos, The generalized Baues problem for cyclic polytopes, I, European J. Combin. 21 (2000), 65-83.

13. Richard P. Stanley, The number of faces of simplicial convex polytopes, Adv. in Math. 35 (1980), 236-238.

14. Richard P. Stanley, Generalized $H$-vectors, intersection cohomology of toric varieties, and related results, in Commutative Algebra and Combinatorics, pp. 187-213. Advanced Studia Pure Mathematics, Vol. 11. North-Holland, Amsterdam, 1987.

15. Richard P. Stanley, Flag-symmetric and locally rank-symmetric partially ordered sets, Electron. J. Combin. 3 \#R6 (1996), 22 pages.

Received December 8, 2000, and in revised form June 21, 2001. Online publication November 2, 2001. 